\title{
Philosophiques
}

\section{Vie et vie pensante dans le Systemfragment que Hegel rédigea lors de son séjour à Francfort}

\section{Laurent-Paul Luc}

Volume 24, numéro 2, automne 1997

URI : https://id.erudit.org/iderudit/027452ar

DOI : https://doi.org/10.7202/027452ar

Aller au sommaire du numéro

Éditeur(s)

Société de philosophie du Québec

ISSN

0316-2923 (imprimé)

1492-1391 (numérique)

Découvrir la revue

Citer cet article

Luc, L.-P. (1997). Vie et vie pensante dans le Systemfragment que Hegel rédigea lors de son séjour à Francfort. Philosophiques, 24(2), 299-311.

https://doi.org/10.7202/027452ar
Résumé de l'article

Au terme de son séjour à Francfort, le jeune Hegel prolonge la tâche qu'il s'était assignée dans ses travaux antérieurs : " penser la pure vie ». Cette tâche, il compte l'assumer en subordonnant la philosophie à la religion. Mais un tel désaveu de la philosophie nous introduit difficilement à l'expérience de l'Esprit dont le Systemfragment fait la « loi vivifiante ". d'utilisation que vous pouvez consulter en ligne.

https://apropos.erudit.org/fr/usagers/politique-dutilisation/ 


\title{
VIE ET VIE PENSANIE DANS IE SYSTEMFRAGMENI QUE HEGEL RÉDIGEA LORS DE SON SÉJOUR À RANCFORT
}

\author{
PAR \\ LAURENT - PAUL LUC
}

RÉSUMÉ: Au terme de son séjour à Francfort, le jeune Hegel prolonge la tâche qu'il s'était assignée dans ses travaux antérieurs : "penser la pure vie ». Cette tâche, il compte l'assumer en subordonnant la philosophie à la religion.

Mais un tel désaveu de la philosophie nous introduit difficilement à l'expérience de l'Esprit dont le Systemfragment fait la "loi vivifiante"

ABSTRACT: At the end of his Frankfurt period, the young Hegel carries on the task that he had undertook in his previous research: "to think pure life". We expect to achieve this task by subordinating philosophy to religion.

But such a denial of philosophy is not an easy introduction to the experience of the Spirit, which is, according to the Systemfragment, the "vivifying law".

L'absence de systématisme ne confine pourtant pas L'Esprit du christianisme à son côté mystique, ni à son côté existentialiste qui " nous fait penser, note Hyppolite, au monde de Kafka ». À travers ces labyrinthes d'une pensée qui peine à déchiffrer les signes d'une bonté infinie, ce sont les portes d'un système que Hegel s'est efforcé d'ouvrir pour y parachever la lente transformation de l'idéal "panique " tubinguien vers ce qui, dès la Vie de Jésus, s'imposait comme le " plan du monde ». Rien ne l'atteste mieux que les textes - datés du 14 septembre 1800 -- les plus accomplis de l'époque francfortoise auxquels Nohl (Hegel's theologische Jugendscriften) donna le nom de Systemfragment. Textes les plus accomplis : on y découvre une remarquable ébauche de systématicité soutenue par une argumentation aussi dense que rigoureuse. Mais aussi textes les plus mutilés : deux fragments discontinus qui constitueraient a moins d'un vingtième v de la deuxième partie d'un ouvrage qui devait être plus volumineux que la version finale de L'Esprit du christianisme. D'où l'intitulé choisi par Nohl.

Une première lecture de ces fragments nous y révèle un Hegel soucieux d'établir une espèce de bilan récapitulatif des étapes qu'il a 
franchies depuis Tübingen. Bilan à première vue ambigu, du moins si l'on tente de l'éclairer à la lumière de la tâche de " penser la pure vie » que s'était assignée $L$ 'Esprit du christianisme. Hegel y dresse en effet un constat de la faillite de la philosophie au profit de la religion : " La philosophie doit cesser avec la religion précisément parce qu'elle est une pensée »( $(348)$. En subordonnant ainsi la philosophie à la religion, en décelant une insuffisance dans toute œuvre de pensée qui a comporte ", écrit-il, " une opposition, pour une part celle de la non-pensée, pour une autre part celle du pensant et du pensé " (§348), Hegel aurait-il renoncé à cette a conscience égale à la vie "dont $L$ 'Esprit du christianisme assurait pourtant que a toutes deux ne diffèrent qu'en ce que la vie est l'être, tandis que la conscience est cet être comme réfléchi " ( $\$ 307)$ ? Comment soutenir maintenant que "la totalité de la nature "doit parvenir jusqu'à a la conscience du monde " (§ 307) si l'énoncé du Systemfragment que nous venons de rapporter proclame l'impossibilité de penser la vie sans détruire son unité ? Énoncé déroutant dont il nous faudra bien peser les termes - à moins de le réduire à une rétractation par Hegel de tout l'effort de pensée qu'il a déployé pour "appliquer les résultats "(Correspondance) de la philosophie. Mais l'hypothèse d'un tel désaveu nous introduirait difficilement à l'expérience de l'Esprit dont le Systemfragment fait la a loi vivifiante " (\$351). Scrutée de près, cette transfiguration ne peut être abordée que comme un affermissement de l'ontologie et de la phénoménologie de la vie esquissées dans les recherches antérieures. En témoignent à elles seules les questions, en apparence secondaires, que Hegel consacre à l'organisation cultuelle de la religion : la construction du temple, la célébration du sacrifice communautaire. Quel sens leur accorde-t-il ?

Le peuple qui accourt au temple hégélien n'y entre pas comme dans un pur lieu, un morceau d'espace déterminé et informe (Gestaltlos) (\$ 349). a Point de réunion (Vereinigungspunkt) d'un grand nombre " $(\$ 350)$, le temple permet la célébration d'une vie liturgique consacrée non pas à un "Dieu invisible » $(\$ 349)$, mais à un Dieu visiblement présent. Une vie liturgique qui doit être belle mais dépouillée de cette a beauté inutile » $(\$ 350)$ qui centre le regard sur l'emplacement du culte ou polarise l'attention, aussi bien intellectuelle qu'imaginative, sur un " Dieu objectif »: " c'est l'essence du service divin ", écrit Hegel, " de relever (Aufzuheben) la considération contemplative ou pensante du Dieu objectif » (§ 350). La liturgie : une ombreuse ferveur? Évidemment non ! Craignant sans doute qu'alors enfermée dans un sombre mutisme, la vie cultuelle se retranche dans ce qu'il évoquait comme " une unité triste et non ressentie „, Hegel corrige sa formulation en ajoutant : « ou plutôt de fondre celle-ci (la considération passive du Dieu objectif) avec la subjectivité des vivants dans la joie, celle du chant et des mouvements corporels, un genre d'extérioration (Aeusserung) subjective qui peut, comme les solennelles exhortations, par le truchement de règles, devenir 
objective et belle, jusqu'à une danse » $(\S 350)$. C'est autour de la spontanéité, de la grâce et de la beauté que se regroupent les traits du culte hégélien.

La célébration du sacrifice communautaire en sera aussi empreinte si elle doit témoigner d'une « réunion (Vereinigung) religieuse " $(\$ 349)$ authentique. En tant que phénomène religieux, la pratique du sacrifice - le Tübinger Fragment s'y était longuement attardé - serait “ sans vie, froide, sans force $(\$ 26)$ si elle ne revêtait pas une figure a légère $n(\$ 25)$ issue de l'esprit d'un peuple rempli a d'émerveillement et de sentiments d'humilité et de reconnaissance » $(\$ 5)$. Autant notre séminariste estimait que " chez un peuple qui a déjà atteint un certain degré d'Aufklärung " (\$ 355) le sacrifice se dégrade en fétichisme et superstition, autant il le considérait comme un des a usages essentiels de la religion " $(\$ 26)$ si on sait l'accueillir - comme il s'en explique, pour la première fois, dans le Systemfragment - comme un moyen qui permet à l'homme de remplir " les conditions négatives de la religion, à savoir d'être libre de toute objectivité absolue, de s'être élevé au-dessus de toute vie finie " $(\$ 349)$. Conditions ne signifie pas ici préparations préalables, mais modalités attachées à l'essence de la religion. Pas plus à Francfort qu'à Tübingen, Hegel ne rabaisse le sacrifice au rang d'un intermédiaire dont dispose la religion pour atteindre ses fins. Si le sacrifice n'est pas, comme la musique sacrée, un “ moyen pur » $(\$ 26)$, il n'en demeure pas moins si essentiel qu'il doit être célébré " périodiquement" (\$ 350). Davantage : il constitue ala seule façon religieuse de se comporter à l'égard d'objets absolus » $(\$ 350)$. On le voit, en reprenant sa méditation tubinguienne du sacrifice religieux, Hegel ne sc contente plus de déclarer que les “ attributs nécessaires des cérémonies religieuses "doivent être tels «que la probabilité qu'elles offrent l'occasion d'un culte idolâtre (Fetischdienste) soit aussi minime que possible; qu'elles ne soient pas constituées de celle manière que ne subsiste que le rougge, le mécanisme et que s'envole l'Esprit »(\$26). Cherchant maintenant à traduire en quoi " les sacrifices [...] appartiennent au système lui-même (de la religion $)$ » $(24)$, il y découvre une impérieuse négativité qui hisse la "réunion religieuse" au rang d'une expérience proprement spirituelle à la condition qu'y soit réussie l'Aufhebung du sensible et de ses prestiges dont l'homme cherche à s'entourer dans un " avoir stable des choses $(\$ 349)$. Avoir à terme irréligieux parce qu'il empêche cette "élévation de la vie finie à la vie infinie »qui spécifie l'essence de la religion. Même a la religion la plus accomplie n n'y échappe pas, elle qui a laisse subsister une certaine hypocrisie due au fait, précisément, que l'on retient pour soi certains biens particuliers " $(\$ 349)$. Comment Hegel nous en divulgue-t-il le secret?

Manifestement - on ne s'en étonnera pas - c'est la référence grecque qui oriente la description hégélienne du sacrifice religieux : " De ses offrandes, il (l'homme) anéantit une partie devant 
la divinité ; le reste, il l'anéantit en lui enlevant le caractère d'être sa propriété privée : en le partageant avec ses amis „( $\$ 50)$. Immolation et consommation, ces deux dimensions du sacrifice en font plus qu'un symbole : un foyer de négativité. Insistons-y. S'il y a une trajectoire spirituelle qu'assume la religion, elle tient tout entière dans cette expérience de la négativité sur laquelle Hegel insiste en qualifiant le sacrifice d'" anéantissement "(Vernichtung), voire même d' anéantissement pour l'anéantissement " (Vernichten um des Vernichtens). À la faveur de cette souffrance de la négativité, la nature et l'homme se spiritualisent : la première s'absout de son objectivité, le second de tous les rapports qui le rivent à sa personnalité, à sa propriété. Fête de l'amour, la vie liturgique ne peut le devenir qu'en célébrant la gloire de la divinité dans la mort, c'est-à-dire au-delà de toute proximité sensible et dans l'anéantissement de toute immédiateté. La description du sacrifice que nous avons citée suscite ce commentaire : "L'inutilité et la superfluité de cet anéantissement sont d'ailleurs une négation supplémentaire de la propriété privée. C'est seulement grâce à cet anéantissement gratuit, grâce à cet anéantissement pour l'anéantissement qu'il rachète les anéantissements qu'il a causés pour ses intérêts particuliers. En même temps il a nié l'objectivité des objets en se livrant à un anéantissement qui ne lui rapporte rien et qui apporte cette négation complète de tous les objets qu'on appelle mort " (\$ 350).

Remarquable approfondissement de la signification du sacrifice religieux mis en débat dès le Tübinger Fragment! Tout autant que la "subjectivité des vivants" à laquelle Hegel vient de référer les formes du culte célébré dans le temple, la mort dans laquelle se nouent l'immolation et la consommation sacrificielle propulse la sphère de la religion dans un "système "qui lui confère sa vérité lorsqu'elle porte témoignage d'un cycle qui libère la " condition humaine " de tout anthropocentrisme : le cycle de la vie et de la mort. La religion n'est religieuse qu'en reliant l'homme à ce cycle. Elle y parvient quand elle enseigne - à la faveur du sacrifice -- que la relation humaine aux objets (destruction et consommation) obéit à un rythme universel, celui-là même de la vie dont Hegel trouve la clef lumineuse dans cette célèbre formule : la vie est a la liaison de la liaison et de la non-liaison (die Verbindung der Verbindung und der Nichtverbindung) "( $(348)$. Mais si c'est là l'essence de toute vie - aussi bien de la vie finie que de la vie infinie - , comment comprendre que Hegel accorde à la religion seule le privilège d'accomplir " l'élévation de l'homme de la vie finie à la vie infinie s? Cette élévation ne pourrait-elle pas être le fait de la vie, de celle qu'on rencontre dans la nature? C'est par le biais de cette question que nous tenterons de circonscrire le plus nettement possible le sens de la formule hégélienne de la vie. Cette question nous est imposée par la désappropriation sacrificielle dont nous pouvons facilement constater qu'elle constitue, aux yeux de Hegel, une sorte de ruse qui permet à la vie de s'assurer d'elle-même : par le sacrifice (immolation des 
animaux, consécration des fruits de la récolte), l'homme religieux ne témoigne-t-il pas que la vie maintient son immortalité à la faveur d'un dépassement de la nature ? C'est précisément cette distance de la vie en elle-même et de la vie telle qu'elle apparaît dans la nature que nous devons d'abord creuser si nous voulons clarifier le geste qui pousse le Systemfragment à réserver à la religion le pouvoir d'élever l'homme de la vie finie à la vie infinie.

La cohérence systématique qui établit cette distance nous est fournie dès la discussion sur le « concept d'individualité » (\$346) sur laquelle s'ouvre le Systemfragment. Concept inconceptualisable, pourrait-on dire, puisque dès qu'on veut concevoir une avie individuelle ", son organisation, on doit la considérer seulement comme relation, c'est-à-dire comme " n'ayant son être que comme léunion (Vereinigung) ", et considérer le reste " seulement dans une opposition "à cette organisation, c'est-à-dire " comme n'ayant son être que par la séparation (Trennung) d'avec celle-ci » (\$346). Bien loin de repousser ces opposés, de les absolutiser, le “ concept d'individualité „ les implique tous deux : une “ vie individuelle » n'est ce qu'elle est, par exemple un homme, que « pour autant qu'il est autre que tous les éléments et (autre) que l'infinité des vies individuelles en dehors de lui ", mais tout aussi bien, " il n'est une vie individuelle que pour autant qu'il est un avec tous les éléments, toute l'infinité de la vie en dehors de lui »( $\$ 346)$. D'où l'expression qui nous est familière : " le Tout de la Vie "(\$346) qu'emploie ici Hegel pour signifier que réunion et séparation, un et multiple doivent être conjugués ensemble pour former un " concept d'individualité " qui ne traduira jamais autre chose qu'une connivence intime entre l'individu vivant et le monde, ou encore, pour nous en remettre à une formule tubinguienne, entre l'hornme et sa relation a à l'égard de ce qui l'entoure et en quoi il vit» $(\$ 4)$. Mais c'est à une pensée qui va bien au-delà de l'aspect social de cet environnement que nous convie le Systemfragment. À vrai dire, cet environnement social qu'ont longuement analysé les travaux de Berne et de Francfort n'a jamais été isolé de la dimension de l'Un-Tout dans laquelle le Tübinger Fragment avait inscrit la " considération de l'homme en général et de sa vie $(\S 4)$. C'est cette dimension ontologique, celle où la considération de l'homme porte sur son être, que vise explicitement le Systemfragment lorsqu'il explore les implications du a concept d'individualité ». Cette visée se signale par l'emploi répété du « $e r$ ist » par lequel Hegel introduit la nécessité de faire appel à une autre logique que celle de la pensée commune - la logique bien connue de l'absolutisation du tout et de la partie - pour penser a l'opposition vivante „ qui lie l'homme à la vie. L'accentuation ontologique de ce lien est bien marquée dans la phrase suivante : “ il est (er ist) seulement dans la mesure où le Tout de la Vie est divisé, lui se trouvant être une partie, tout le reste l'autre partie ; il est (er ist) dans la mesure seulement où il n'est pas une partie, et que rien n'est séparé de lui » $(346)$. 
Ce rapport ontologique qui est celui de la vie et des vivants, nous pouvons l'aborder selon deux perspectives différentes. L'une consiste à penser la vie comme “vie indivise ». Selon ce point de vue, le rapport vie-vivant en est un de manifestation. Les vivants apparaissent comme étant des " extériorations "(Aeusserungen), des " présentations "(Darstellungen) de la vie. Ici, nous sommes en présence de la vie comme d'une vie immortelle, encore que la fluidité de la vie soit cristallisée " en points stables, subsistants, fixés, en individus " ( $\$ 346)$. Ce travail de cristallisation est aussi précieux que décisif : il s'agit de diviser les " extériorations " de la vie en individualités. Cette division est l'œuvre de la réflexion. Sclon la scconde perspective, il ne s'agit plus de penser la vie à partir de son indivision, mais à partir de ce que Hegel appelle un "vivant présupposé, à savoir », écrit-il, "nous qui considérons " (\$ 346). Comment apparaît alors la vie? Puisque nous choisissons de considérer la vie en spectateurs, elle ne nous apparaît pas seulement comme une vie infinie située " en dehors de notre vie limitée ", mais aussi comme une vie figée que notre réflexion pose à la fois " en tant que multiplicité infinie d'organisations, d'individus (et) en tant qu'unité, un tout unique organisé, séparé et réuni "(\$346). À proprement parler, cette vie n'est pas la vie elle-même, mais la nature, la vie en tant qu'elle est posée par la réflexion. « Elle est une position de la vie, car la réflexion a introduit dans la vie ses concepts de relation et de séparation (Trennung), de l'individu, existant pour soi, et de l'universel, du relié, donc du limité et de l'illimité, et par cet acte de poser, elle a transformé la vie en nature "(\$346-347). Difficile d'entendre dans cette conception de la nature les échos qu'évoquaient les textes antérieurs lorsqu'ils saluaient le génie d'une Grèce spontanément accordée à un monde qui s'offrait à elle pour ainsi dire naturellement. Le " nous nous sommes éloignés de la nature "de Tübingen $(\S 355)$ trahissait-il une conception de la modernité qui ne réussira à se réconcilier avec la nature qu'après avoir maîtrisé ce qui, au fond, n'est que l'œuvre du Moi se posant en tant que réflexion? Cette hypothèse paraîtrait justifiée s'il fallait n'adopter que la seconde perspective : pour s'approcher de la nature, aucune situation ne paraît plus enviable que celle du spectateur qui l'aborde à la manière d'un monde extérieur dont il pose lui-même les lois. Mais cette situation, le Systemfragment est loin d'y souscrire même s'il déclare que la nature y est considérée de la façon " la plus digne " : « cette manière de poser, de considérer ", écrit Hegel, « est unilatérale »(\$347). Comment nous l'expose-t-il ?

En nous rappelant la nécessité d'une unité absolue qui résorbera " ce fait que le Vivant est, dans son être, fragmentaire (dieses Teilsein den Lebendigen ) ( $(348)$ ! Chaque fois que nous adoptons la perspective de la réflexion pour tenter de surprendre comment la vie individuelle peut être à la fois niée et affirmée, nous nous rendons compte, par la raison, de la partialité de l'attitude réflexive. Davantage : c'est le vivant lui-même qui a sent "comme une 
incomplétude de la vie : a quand elle se rapporte à la nature, la vie examinatrice, pensante (das Natur betrachtende, denkende Leben) sent (fihlt) encore cette contradiction, cette opposition unique existant entre elle-même et la vie infinie » (\$347). On saisirait mal la portée de cette méditation si on décelait sous l'étrange équivalence raison-vie pensante établie par Hegel un souci de rejeter le travail de la réflexion. Hegel a beau écrire que « la nature n'est pas la vie, mais la vie solidifiée par la réflexion ", l'incomplétude soulignée dans cette phrase ne pointe aucunement un échec de la réflexion. Elle signifie que du point de vue réflexif et précisément parce qu'il laisse régner dans la nature a la réunion (Vereinigung) du fini et de l'infini et la séparation (Trennung) de ceux-ci " ( $\$ 347)$, on assistera à ce prodige : la division de l'indivisible, mais on ne verra jamais s'accomplir l'indestructible unité du monde. Ce besoin d'accéder à cet accomplissement s'impose avec une telle force que pour le satisfaire, l'homme doit dépouiller la vie de tout " ce qui passe ", en abstraire un Vivant pur qu'il nomme Dieu et auquel il se lie au-delà de toute "relation pensée ": "La Vie pensante (Denkende Leben) fait ressortir (hebt heraus) hors de la forme finie, de ce qui est mortel et passager, hors des éléments qui s'opposent et se combattent infiniment, le Vivant, le Vivant qui est libre de ce qui passe, elle fait ressortir la relation dépouillée de ce qu'il y a de mort et de mourant dans la multiplicité, elle fait ressortir non pas une unité, une relation pensée mais la Vie infinie, entièrement vivante et toute-puissante, et la nomme Dieu, et elle n'est jamais pensante (mimmer denkend) ou examinatrice (betrachtend) parce que son objet ne porte en lui rien de réfléchi, rien d'inanimé n (\$347).

Par cette distinction entre la "Vie pensante " et la "Vie pensante " qui “ n'est jamais pensante », Hegel oriente l'apaisement du désir de laisser s'accomplir l'unité du monde dans le sens d'une "élévation de l'homme non du fini à l'infini, car ces deux termes ne sont que des produits de la simple réflexion, et, comme tels, leur séparation (Trennung) est absolue, mais de la vie finie à la vie infinie ( $\$ 347)$. Cette élévation, écrit-il, "est religion ». Cet appel, ćclairons-le à la lumière d'un passage de L'Esprit du christianisme où nous voyons la croyance d'un Jésus en son Dieu s'opposer radicalement à celle des Juifs. Alors que ces derniers croient en leur Dieu comme à un contraire du fini, Jésus, lui, appréhende dans la Vie pure non une unité abstraite mais a la source d'où découle [...] toute figure de la vie limitée » $(\$ 303)$. C'est cette source qui revient dans le Systemfragment sous le vocable "Vie infinie ". Même nommée Dieu, il ne peut s'agir d'une vie extérieure à ce qu'elle réunit. Et pour bien souligner que cette Vie n'unifie pas la nature du dehors, mais qu'elle réside tout entière dans la réunion de ses extériorations et de son unicité, Hegel fait remarquer qu' on peut appeler la Vie infinie un Esprit, par opposition à la pluralité abstraite, car l'Esprit est l'unicité vivante de la diversité en opposition avec cette diversité en tant que celle-ci est sa figure [laquelle constitue la diversité inhérente 
au concept de la vie], non en opposition avec la diversité en tant qu'elle est séparée (getrennte) de l'esprit, morte, simple pluralité ; car alors l'Esprit serait la simple unité qu'on appelle loi et un simple pensé, inanimé. L'Esprit est la loi vivante en réunion (in Vereinigung) avec le divers, qui est alors un animé » $(\$ 347)$. Ces lignes méritent une attention particulière : elles tracent la constellation spirituelle où gravitent philosophie et religion pour dessiner le cercle de a l'unité vivante de la diversité ». Le passage cité s`interroge sur la possibilité de traduire ce cercle dans le " concept de la vie ". Quelles en sont les chances?

Un tel concept devrait, selon Hegel, pouvoir permettre de penser une unité qui porte en elle une opposition. Une “ unité vivante "qui ignorerait l'opposition - une unité du type de celle que $L$ Esprit du. christianisme décèle sous la conception d'un a Dieu isolé » : un Dieu pur esprit, infiniment infini - ne serait qu'une " simple unité " dont elle n'aurait de l'unité que le nom puisqu'elle ne serait qu'un opposé, l'opposé de la pluralité. C'est à cette unité inerte, abstraite que conduit la réflexion impuissante, pour cette raison, à saisir une unité spirituelle, une unité reliée à la pluralité, non pas comme à une pluralité morte, mais comme à une pluralité qui est " sa figure ». Le divers apparaîtrait-il alor's comme "entièrement relié "à l'unité ? Exiger cette liaison ne serait que confirmer l'emprise de l'abstraction. Cette exigence s'avère même trop spiritualisante. Si le divers est considéré comme n'étant plus séparé de l'unité, “quelque chose est exclu : la matière morte, et il en résulte une incomplétude et une opposition. En d'autres termes, quand on pose le divers uniquement dans sa liaison avec l'Esprit, [...], l'opposition elle-même est exclue " (\$ 348). Autant dire que s'il doit y avoir un " concept de la vie ", il doit être tel qu'en lui la vie " ne peut être considérée (betrachtet) seulement comme réunion (Vereinigung), relation mais doit en même temps être considérée comme opposition ( $\$ 348$ ). Faudrait-il dire alors que la fonction unifiante assurée par l'unité vivante oblige à considérer cette dernière comme une liaison de la réunion et de l'opposition? Cette formulation ne traduirait pas mieux la mouvance de la vie puisqu'elle privilégie la liaison à son contraire. Aussi l'expression la mieux accordée à la vie s'énonccrait-elle de la manière suivante : "la liaison de la liaison et de la non-liaison ". Mais même cette expression n'évite pas les pièges de la réflexion : on peut toujours lui opposer l'objection soulevée contre la formulation précédente. Et rien n'empêche de continuer ainsi à l'infini. " C'est-àdire ", poursuit notre texte, "chaque expression est un produit de la réflexion, et par suite on peut montrer de chacune en tant qu'elle est quelque chose de posé, que, parce que quelque chose est posé, en même temps quelque chose d'autre n'est pas posé, est exclu » ( $\$ 348$ ). Le “ concept de la vie»? Un interminable chassé-croisé ! Aussi artificiel qu'inutile, voire même profanateur, s'il est vrai, comme insiste L'Esprit du christianisme, que la vie est ce mystère sacré qui se dérobe à tout discours. Hegel ne s'émerveillait-il pas devant l'attitude 
pudique qu'adoptaient les initiés aux mystères éleusiniens : des « manifestations du Dieu à Éleusis, personne n'était exclu ; il était seulement interdit d'en parler, car des paroles les eussent profanées " $(\$ 257)$. N'écrivait-il pas aussi que « la vie ne se saisit que par la vie " $(\$ 319)$, que la vie ne peut être vécue que comme Esprit : a là où il n'y a pas d'âme, pas d'esprit, il n'y a non plus rien de divin ; dans l'être qui se sent toujours déterminé, comme faisant ou subissant toujours ceci ou cela, agissant de telle ou telle manière, dans cette abstraction, il n'y a pas séparation du limité et de l'esprit, mais ce qui demeure n'est bien plutôt que l'opposé du vivant " (§303). En soustrayant la vie aux artifices de la réflexion, le Systemfragment réaffirme que le sens de l'être de la vie s'accomplit comme Esprit. À ce titre, la vie est impensable.

Comment interpréter cette conclusion que fait déjà ressortir $L ' E s p r i t$ du christianisme en creusant une tension insurmontable entre la vie et la conceptualité : Begreifen ist beherrschen? Si Hegel écrivait que la " simple réflexion n'est pas apte à exprimer le spirituel selon l'esprit " $(\S 306)$, si toute thèse “ exprimée dans la forme de la réflexion » lui apparaissait « insensée (widersinnig)" ( $(306)$, était-ce, en fin de compte, parce que toute démarche réflexive, essentiellement confinée à une visée de l'identité, masque, à ses yeux, la luxuriance foisonnante de la vie, en nivelle les différences? La vie : une fragmentation? Le Systemfragment n'est pas loin d'adhérer à une vie qui serait pure différence lorsque définissant la vie comme "la liaison de la liaison et de la non-liaison ", il en fait un mouvement infini de différenciation que la réflexion ne peut tenter d'épouser qu'à travers un passage incessant d'un opposé à un autre : de la pensée à la non-pensée, du pensant au pensé, du subjectif à l'objectif, du limité au limitant, du vivant au mort, du fini à l'infini. Comme si la célèbre formule célébrait une vie sauvage, rebelle à toute clôturation. Pourtant, cette compréhension d'une vie inclôturable, indivisible, le Systemfragment l'attribue à la vie finie (ou à la réflexion de la vie infinie) vouée à une errance, à une "impulsion "qui la fait dériver sans cesse d'une expression à l'autre, d'un extrême à l'autre. C'est précisément « à cet être-poussétoujours-plus-loin (Fortgetriebenwerden) sans point de repos (Ruhepunkt) "qu'il faut, selon Hegel, " une fois pour toutes obvier» (\$348). Que la vie soit impensable n'est donc pas imputable au motif de la différence, mais à ce que L'Esprit du christianisme appelle une inaptitude de la réflexion à " exprimer le spirituel selon l'esprit ". C'est-à-dire dans un " point de repos " d'où la Vie puisse laisser advenir le déploiement de sa présence en échappant à sa fragmentation. On y parvient, selon Hegel, quand on quitte la philosophie pour la religion : « Ce fait que le Vivant est, dans son être, fragmentaire se supprime dans la religion, la vie limitée s'élève jusqu'à l'infini ; et c'est uniquement parce que le fini est lui-même vie, qu'il porte en lui la possibilité de s'élever jusqu'à la vie infinie. La philosophie doit précisément pour cela cesser avec la 
religion „(§ 348). Avant de préciser en quoi la religion ménage l'accès à la liaison vivante accomplie, Hegel s'attache à délimiter la tâche de la philosophie.

Cette tâche est essentiellement critique. Conçue comme une “ activité de la raison " (§348), la philosophie peut bien conduire à une élévation à l'infini, mais jamais autrement que comme un idéal qu'elle pose au-delà de sa propre sphère de pensée. Elle ne fait fleurir aucune danse, elle « a à montrer dans tout fini la finitude et à exiger la complétude de celui-ci par la raison, à connaître en particulier les illusions causées par son propre infini, et ainsi à poser le véritable infini hors de sa sphère " (\$348). Si la philosophie demeure hors du domaine de l'infini véritable, si elle ne conduit qu'à un faux infini, si elle est en proie à un mouvement " sans point de repos ", c'est qu'elle ne peut penser le fini sans lui opposer un infini qui le limite et le dépasse. Davantage : sans faire de cette opposition un absolu. N'étant qu'un dehors, un au-delà du fini, son infini est donc posé comme ne pouvant être posé par elle. Si le fini y atteint sa complétude, ce ne peut être qu'à la faveur d'une unité qui ne sera jamais qu'une synthèse pensée. Autrement dit : "un produit de l'entendement (Verständiges), quelque chose de réfléchi » et dont l'a unique caractère pour la réflexion est qu'il est un être en dehors de la réflexion „ (\$348). La religion, quant à elle, conduit au véritable infini parce qu'c elle ne pose pas l'être de l'infini comme un être produit par la réflexion, comme un être objectif ou subjectif ». L'être auquel elle conduit est une liaison vivante authentique dans la mesure, insiste encore Hegel, où elle ne sombre pas dans ce mouvement sans fin où la philosophie « ajoute au limité le limitant, reconnaît aussitôt celui-ci comme un posé, même comme un limité et recherche de nouveau ce qui la limite et ainsi de suite jusqu'à l'infini » (§348). À s'en tenir à la description de ce cercle contradictoire dans lequel la fin du premier feuillet du Systemfragment enferme " l'activité de la raison ", on pourrait croire que l'élévation religieuse à la vie infinie s'accomplit dans une démarche réservée au pur sentiment. D'autant plus facilement que c'est le vivant, nous a dit Hegel, qui " sent "l'incomplétude de la vie finie. En critiquant une Vie pensante qui ne serait que pensante, Hegel ne nous a-t-il pas laissé entendre que c'est en deça de tout dualisme de la réflexion, en deça de tout "produit de l'entendement", de tout processus objectivant, que se nourrit la quête religieuse de a l'être de l'infini $₫$ ? Cet être ne s'authentifierait dans aucune réflexion, dans aucune objectivité. Mais cette interprétation ne rend pas justice à la teneur de la religion telle que nous la présente Hegel. Retrancher l'expérience religieuse dans une pure subjectivité, ne serait-ce pas ranimer l'opposition produite par la réflexion ? Non qu'il faille, pour Hegel, annuler l'opposition du subjectif et de l'objectif, mais maintenir ces opposés dans leur relation en les laissant jaillir de leur " unité vivante " comme de leur " point de réunion ». Ce privilège 
est réservé à la religion. Considérons sous quel jour elle est pensée par Hegel.

"Le sentiment du divin », lisons-nous au début du second feuillet du Systemfragment, a l'infini senti (gefühlt) par le fini n'atteint la complétude que dans la mesure où la réflexion s'y introduit et demeure en lui » (\$349). Le sens de cette phrase est clair. L'élévation religieuse à la vie infinie passe par le sentiment mais ne s'y confine pas, sous peine de se dégrader en sentimentalisme. Elle exige que le sentiment soit réfléchi. En quel sens? Il ne peut s’agir évidemment - Hegel ne manque pas de le rappeler - de cette réflexion mise en ceuvre dans une vie qui n'est que vie pensante, c'est-à-dire de cette réflexion qui n'est jamais qu'une réflexion sur le sentiment - a une réflexion séparée par le sentiment séparé » (\$ 349) - , une réflexion qui ne le reconnaît que "comme quelque chose de subjectif "et qui produit ce que Hegel appelle ironiquement " seulement une conscience du sentiment (nur ein Bewusstsein des Geefühls) "(\$349). Dans la sphère religieuse, sentiment réfléchi veut dire sentiment se réfléchissant. Comme une subjectivité dans une objectivité. Mais à la condition de ne pas en faire les extrêmes d'une "opposition cristallisée » (\$351): dans la réflexion du sentiment, subjectivité et objectivité se recoupent, chacune appelant l'autre - et comme son autre, de telle sorte que pas plus que la a pure subjectivité ", la " pure objectivité " n’y a de place. Ainsi, lorsqu'un peuple bâtit une " maison de Dieu », le temple peut bien se dresser comme un " centre objectif ", mais on ne verrait pas comment Dieu y habite si on en faisait l'affaire de ce que le Tübinger Fragment décrivait sous le vocable de « religion objective ». Si on le ramenait, autrement dit, à une objectivité d'entendement : à un simple morceau d'espace, à une « pure objectivité spatiale »qui ne retourne jamais à la génialité artistique de tout un peuple qui s'y est pourtant manifesté en apaisant le combat du ciel et de la terre, de l'esprit et de la matière, dans un « point de réunion ». En ce point, c'est “ l'Être infini dans l'espace incommensurable»qui se concentre. Sans chercher dans le fini autre chose que l'élément de son développement. Jamais un support à sa présence. Plus que l'image d'un cercle, c'est celle d'une fusion (Verschmelzen) $(\$ 350$ ) de la subjectivité et de l'objectivité qui fait irruption ici. Pour saluer " l'unité vivante "d'un monde dont la beauté est inaccessible à la a simple réflexion». C'est son aveuglement à l'cEsprit du Tout"(\$347), son inhabilité à retrouver " l'être de l'infini "à la fois même et autre que décrivent ces lignes célébrant, à même la vue du temple, la transfiguration des choses opérée par la religion :

la pure objectivité figurée (gestaltete) est en même temps ce qu'elle doit être à la faveur de la subjectivité qui lui est liée, non pas une objectivité effective, mais seulement une objectivité possible : elle peut être pensée en tant que telle (comme objectivité), mais ce n'est pas nécessaire parce qu'elle n'est pas purement objective [...]. L'Être infini dans l'espace incommensurable est en même temps dans un espace déterminé, un peu 
comme il est dit dans l'hymne : celui que tous les cieux des cieux ne peuvent contenir, voilà quil repose dans le sein de Marie. (§ 341)

Allégation imprudente? Subversive, dirions-nous plutôt, s'il est vrai que, dès Éleusis, la figuration de l'incommensurable a toujours été soustraite - et radicalement - à la religion chrétienne dont L'Esprit du christianisme a dénoncé un trop grand attachement à une a opposition au sein du divin, présent dans la conscience el qui ne peut jamais l'être dans la vie ». Religion trop déchirée entre l'action divine et l'action dans le monde, écrivait encore Hegel, pour les laisser" se fondre en une seule (in Eins zusammenschmelzen) " (\$341)! La méditation sur l'élévation religieuse de la vie finie à la vie infinie n'a pas fait oublier l'indignation soulevée par le christianisme : il enseigne à " dépendre d'une essence absolument étrangère qui ne peut devenir homme, ou, au cas où elle serait devenue telle (donc dans le temps), dans cette réunion (Vereinigung) aussi resterait un être absolument particulier, seulement un absolu $(\$ 351)$. Cette critique ne serait pas nouvelle si elle ne considérait "l'esclavage sous I'Objet immensément grand " (\$351) propagé par le christianisme comme étant celui de l'époque présente qui ne peut et jusque chez ses philosophes (nommément Fichte) dépasser la finitude que dans une infinité séparée du fini. "Phénomène du temps ", écrit Hegel, que celui de la " séparation (Trennung) infinie ", de l'opposition " de l'absolument fini et de l'absolument infini " (\$351). Introduite comme le ressort d'une élévation à la vie infinie, cette séparation et cette opposition transforment la sphère religieuse en une évasion de la vie fínie qui comme telle doit être traitée comme « ignoble et abjecte (\$ 351).

Si on s'attarde aux voies modernes (philosophique et religieuse) d'une élévation à la vie infinie, on remarque, d'après Hegel, qu'elles reposent sur une opposition fini-infini dont la signification est double. Elle signifie " un vol (Schweben) du Moi au-dessus de toute nature, ou la dépendance, ou mieux la relation à une essence qui est au-dessus de toute nature ". Dans le premier cas, on voit le Moi " se poser comme pur Moi, au-dessus des ruines de ce corps el des soleils qui illuminent, au-dessus des myriades de corps célestes "; dans le second, on le voit « craindre un Dieu qui est infiniment élevé audessus du ciel de tous les cieux, au-dessus de toute liaison, de toute appartenance, planant tout-puissant au-dessus de loute nature $(\$ 351)$. Philosophie et religion se rejoignent dans la sauvegarde d'une « unité suprême ». Mais que celle-là cherche cette unité " du côté de la subjectivité " en l'investissant d' " autonomie (Selbständigkeit) " ou que celle-ci la trouve dans « un objet étranger, lointain, inaccessible ", l'une et l'autre " se combinent " pour faire de la condition humaine un état où " l'homme doit paraître asservi ". Idéalisme et théisme sont la forme convenue du mépris de la vie. Lisons Hegel : “Dans les deux cas, l'élévation de la vie finie à la vie infinie ne pourrait être qu'une élévation au-dessus d'une vie 
finie ; l'infini est le plus complet, dans la mesure où il serait opposé à la totalité, c'est-à-dire à l'infinité du fini, non dans la mesure où cette opposition serait supprimée dans une belle réunion (Vereinigung)"(\$351). Que dans une religion accomplie, toute opposition se résolve en beauté, n'est-ce pas ce que suggère Hegel lor'squ'il avoue sa préférence pour une religion belle qu'il éloigne de toute religion où on en vient à se fixer à un Dieu transcendant ou à s'exiler dans un Moi sans monde, celui qui a a toute chose $[. .$.$] sous$ ses pieds »? Toute autre religion que la religion belle " peut être sublime, écrit-il, et effroyablement sublime, mais elle ne peut être d'une belle humanité ". Cet aveu s'accompagne d'une prise de position trop catégorique pour qu'on n'y soupçonne pas une difficulté que Hegel est encore loin d'avoir maîtrisée. La voici : la religion du sublime serait ce qu'il y a a de plus digne, de plus noble, si la réunion avec le temps (Vereinigung mit der Zeit) était ignoble et abjecte»(§351).

Faculté de théologie, d'éthique

et de philosophie

Université de Sherbrooke 